\section{Cost-effective treatment of acute myocardial infarction with sirolimus-eluting stents}

Bare-metal stent implantation with abciximab pretreatment is a recommended reperfusion strategy for acute ST-segment elevation myocardial infarction (STEMI). Although sirolimus-eluting stents reduce the need for target-vessel revascularization, the lack of randomized trials and greater procedural costs currently restricts their use. Valgimigli and colleagues proposed that replacing abciximab with the cheaper drug tirofiban might compensate for the high cost of using sirolimus-eluting stents.

The investigators designed a prospective single-blind randomized controlled study to compare the clinical and angiographic impact of single high-dose bolus tirofiban plus sirolimuseluting stenting, with abciximab plus bare-metal stenting, in patients with STEMI. The study included 175 patients with a median age of 63 years diagnosed with STEMI or presumed new left-bundle-branch block. Patient outcomes were recorded after 30-day and 8-month intervals for 74 patients treated with single highdose bolus tirofiban plus sirolimus-eluting stent and 74 patients treated with standard-dose abciximab plus bare-metal stents.

While the overall incidence of death or reinfarction was similar for the two regimens$13 \%$ in the tirofiban plus sirolimus-eluting stent group and $17 \%$ in the abciximab plus bare-metal stent group (hazard ratio, $0.71 ; 95 \% \mathrm{Cl}, 0.34-1.5$; $P=0.39$ ) - the need for target-vessel revascularization was significantly lower in the tirofiban group (7\% vs $20 \%, 95 \% \mathrm{Cl}, 0.12-0.77 ; P=0.01$ ), and less in-lesion binary restenosis was reported in these patients ( $9 \%$ vs $36 \%, P=0.002)$.

Valgimigli et al. conclude that tirofiban treatment with sirolimus-eluting stent implantation is a promising lower-cost treatment strategy for STEMI, incorporating unrestricted use of sirolimus-eluting stenting.

Original article Valgimigli et al. (2005) Tirofiban and sirolimus-eluting stent vs abciximab and bare-metal stent for acute myocardial infarction. JAMA 17: 2109-2117

\section{Trends in coronary heart disease among British men}

Since the late 1970s, the incidence of major coronary events has steadily declined in the
UK. Much less is known about the incidence of angina in the population as most cases of angina are diagnosed and managed in primary care.

The British Regional Heart Study is a prospective study of 7,735 men aged $40-59$ years at baseline (1978-1980), who were randomly selected from one general practice in each of 24 British towns. Lampe et al. compared incidence rates of first-diagnosed angina, first-diagnosed coronary heart disease, and total incidence of major coronary events in middle-aged men included in the British Regional Heart Study. Participants were followed over 20 years through NHS central registers and regular reviews of medical records in general practices.

From 1978 to 2000 , while the rate of all major coronary events declined by an average of $3.6 \%$ per year, the rate of first-diagnosed angina increased by an average of $2.6 \%$ per year, and these opposing trends resulted in no apparent change in the overall incidence of diagnosed coronary heart disease. The reported increase in the incidence of angina might reflect improvements in diagnosis and increased availability of diagnostic investigations for angina-like pain symptoms, rather than a genuine increase in disease occurrence.

This study emphasizes the ongoing need for treatments and services for coronary heart disease, particularly cases of new or suspected angina.

Original article Lampe et al. (2005) Trends in rates of different forms of diagnosed coronary heart disease, 1978 to 2000: prospective, population based study of British men. BMJ330: 1046

\section{Ventricular assist normalizes inducible nitric oxide synthase and reduces apoptosis in heart failure}

Nitric oxide is proposed to contribute to heart failure and recent studies have revealed high levels of inducible nitric oxide synthase (iNOS) in the myocardium of failing hearts. Patten et al. investigated the effect of ventricular assist device (VAD) therapy on iNOS expression and apoptosis in the myocardium of patients with end-stage heart failure due to left-ventricular systolic dysfunction.

Myocardial tissue samples were analyzed from 9 patients at the time of heart transplantation (transplant group), 10 patients during VAD placement (pre-VAD group) and 11 patients at 